\title{
Dificuldades Iniciais no Aprendizado do Exame Físico na Percepção do Estudante
}

\section{Initial Difficulties in Learning the Physical Exam According to the Student's Perception}

\author{
Gilka Paiva Oliveira Costa ${ }^{I}$ (D) \\ Kaio Alighieri Nunes de França ${ }^{I}$ (DD \\ Maria Albertina Leite Santos ${ }^{I}$ (iD \\ Janaína Guerra Guilherme ${ }^{I}(\mathbb{D})$ \\ José Givaldo Melquiades de Medeiros ${ }^{I}$ (iD \\ Estácio Amaro da Silva Júnior ${ }^{I}$ (iD
}

\section{PALAVRAS-CHAVE}

- Exame Físico.

- Estudantes de Medicina.

- Faculdades de Medicina.

- Educação Médica. ser geradoras de insegurança e ansiedade, entre as quais se destacam as primeiras aulas práticas com pacientes que tradicionalmente ocorrem na disciplina de Semiologia Médica. A realização do primeiro exame físico com um paciente real é uma potencial circunstância geradora de estresse para os que dão seus primeiros passos na construção de habilidades indispensáveis à carreira médica. A forma de lidar com o estresse advindo desses encontros é bastante individual e relaciona-se com diversos fatores inerentes ao estudante e ao modelo de ensino adotado. O presente estudo propõe-se a identificar as principais adversidades relatadas pelos alunos na sua iniciação ao exame físico. Método: Para tanto, realizou-se um estudo exploratório, com abordagem qualitativa, executado durante as atividades do Módulo Horizontal Básico 4 (MHB4), o qual está inserido na grade curricular do quarto semestre do curso de Medicina da Universidade Federal da Paraíba. Uma amostra de 35 estudantes foi dividida em seis grupos focais. A coleta de dados foi realizada na oitava semana do semestre letivo e ocorreu simultaneamente em todos os grupos. A discussão iniciouse por meio da pergunta norteadora "Quais são as dificuldades encontradas durante o exame físico do paciente?”. Procedeu-se, então, à gravação das falas dos alunos, com posterior transcrição, na íntegra, de todo o material textual, o qual foi submetido à análise de conteúdo por meio do método preconizado por Bardin. Resultados: As falas dos alunos foram elencadas em cinco categorias nomeadas como: insegurança e inexperiência; submissão do paciente; sensação de estar incomodando o paciente; direcionamento de gênero; escolha do paciente por conveniência. A partir dessa análise foi possível observar que a realização do primeiro exame físico é um evento gerador de grande estresse emocional para os estudantes. Questões como a insegurança, a inexperiência e a sensação de estar utilizando o paciente como um objeto são citadas como fatores que geram desconforto nos estudantes e dificultam seu aprendizado. Conclusões: As percepções dos estudantes são de insegurança, inexperiência e invasão da privacidade de um paciente que se encontra numa situação de passividade diante da situação. Nesse contexto, o enfrentamento das dificuldades toma proporções maiores e irrealísticas no aprendizado do exame físico. 


\section{KEY-WORDS}

- Physical Examination.

- Medical Students.

- Medical School.

- Medical Education.

\section{ABSTRACT}

Introduction: During Medical School, the student faces several situations that can generate insecurity and anxiety, and among them, the one that stands out is the first practical class with patients, which traditionally occur during the Medical Semiology course. Performing the first physical examination in a real patient is a potential stress-inducing situation for those who take their first steps towards building the necessary skills for a medical career. The way one deals with the stress generated by these situations is quite individual and is related to several factors inherent to the student and to the teaching model adopted. The present study aims to identify the main adversities reported by students in their initiation to physical examination. Method: An exploratory study with a qualitative approach was carried out during the activities of the Basic Horizontal Module 4 (MHB4), which is part of the curriculum of the fourth semester of the Medical School at Federal University of Paraíba. A sample of 35 students was divided into 6 focal groups. Data collection was performed in the eighth week of the semester and occurred simultaneously in all groups. The discussion began with the guiding question "What are the difficulties faced during the physical examination of the patient?". The student's speeches were recorded and then transcripted in full and the material was submitted to content analysis using the method recommended by Bardin ${ }^{1}$. Results: The students' statements were listed into 5 categories: insecurity and inexperience; patient's submission; feeling of bothering the patient; gender targeting; selection of the patient for convenience. Based on this analysis it was possible to observe that the performance of the first physical examination is an event that causes much emotional stress to students. Issues such as insecurity, inexperience and the feeling of using the patient as an object are mentioned as factors that causes discomfort to students and hinder their learning process. Conclusions: The students' perceptions are those of insecurity, inexperience and invasion of the patient's privacy, who is in a passive situation. In this context, the process of facing the difficulties takes on bigger and unrealistic proportions in the learning process of physical examination.

Recebido em 22/10/19

Aceito em 26/11/19

\section{INTRODUÇÃO}

Ao iniciar o curso de Medicina, uma das maiores expectativas do ingressante é o primeiro contato com o paciente. Esse contato inicia-se, tradicionalmente, na disciplina de Semiologia Médica, na qual o aluno começa a conhecer as técnicas para realização da anamnese e do exame físico ${ }^{1}$.

Diante de um paciente, apesar dos avanços tecnológicos, habilidades para colher a história clínica, realizar exame físico e comunicar-se com o paciente permanecem como ferramentas mais importantes para colher dados e indicar hipóteses diagnósticas ${ }^{2}$.

Entretanto, ao iniciar sua prática em exames clínicos, o aluno encontra inúmeras dificuldades. Isso ocorre porque, nesse momento, integra-se o paciente como mais um participante no processo ensino-aprendizagem. Agregado, ele transforma a relação dual estudante-professor numa tríade, estudante-professor-paciente, despertando no estudante sentimentos variados e o forçando a se adaptar à nova realidade ${ }^{3}$.

A forma de lidar com esses primeiros encontros, tidos como estressantes, é bem individual; e as reações a eles, por demais diversificadas, considerando-se aí elementos como a imaturidade do aluno e as circunstâncias inerentes ao modelo de ensino囚. Sendo assim, os estressores podem determinar comportamentos inadequados, contribuindo para mais uma fonte de estresse para alunos do curso médico, quando da construção de habilidades necessárias à prática da Medicina.

A graduação em Medicina tende a exigir do aluno um complexo preparo técnico-relacional, desenvolvido a partir de estudos teóricos e experiências assistenciais supervisionadas ${ }^{5}$. Ao longo do curso, as habilidades vão sendo construídas a partir de como as vivências vão sendo experimentadas e superadas, o que está diretamente relacionado à capacidade de resiliência do estudante, assim como é fonte de demanda contínua que também, de acordo com o enfrentamento e superação, tem relação direta com a ocorrência de burnout ${ }^{6}$.

Considerando que o reconhecimento das dificuldades vivenciadas pelos acadêmicos pode favorecer o planejamento de estratégias que promovam a construção de habilidades e atitudes positivas na formação médica, este trabalho se propõe a identificar as principais adversidades percebidas pelos alunos na sua iniciação ao exame físico.

\section{MÉTODOS}

Trata-se de um estudo exploratório, com abordagem qualitativa, realizado no quarto período do curso médico da Universidade Federal da Paraíba (UFPB), o qual é organizado dentro de uma estrutura modular. Nesse período, os alunos estão em atividades introdutórias da Semiologia Médica e cursando o Módulo Horizontal Básico 4 (MHB4), que tem como base a psicologia médica. Esse módulo trabalha com o estudante de Medicina o reconhecimento das manifestações emocionais do paciente, das suas próprias reações e sofrimentos, diante da especificidade da profissão médica ${ }^{7}$.

A coleta de dados foi realizada na oitava semana do semestre letivo e ocorreu simultaneamente em todos os grupos, sendo cada um mediado por um monitor da disciplina. Formaram-se seis grupos focais, sendo cinco compostos por seis alunos e um por cinco alunos, totalizando, assim, uma amostra de 35 alunos. Foram critérios de seleção dos participantes: estar matriculado nos módulos MHB4 e Semiologia Médica, bem como ter iniciado as atividades de exame físico, o que subsidiou as discussões 
do presente trabalho. No início da sessão, que teve duração média de 60 minutos, fez-se a pergunta norteadora "Quais são as dificuldades encontradas durante o exame físico do paciente?”. Procedeu-se, então, à gravação das falas dos alunos, com posterior transcrição, na íntegra, de todo o conteúdo.

Todo o material produzido das transcrições foi agrupado como texto único e submetido à análise de conteúdo por meio do método preconizado por Bardin ${ }^{8}$, cujas etapas se dividem em: pré-análise, que compreende a leitura geral do material escolhido para análise, a fim de estabelecer indicadores para interpretação dos dados coletados já transcritos; exploração do material, cujas partes das falas são recortadas em unidades de registros, ou seja, em categorias primeiramente iniciais (subjetivas dos autores), depois intermediárias e, por último, reorganizadas em categorias finais; e interpretação, a fim de entender as manifestações contidas no material coletado ${ }^{9}$.

A partir da categorização, foi iniciada a análise. As falas separadas em categorias, de acordo com a semelhança de conteúdo entre elas, possibilitaram respostas à pergunta norteadora, a fim de compreender os significados do contexto estudado.

Para a apresentação dos trechos das entrevistas, nos resultados do estudo, os participantes foram mantidos em anonimato e assinaram o Termo de Consentimento Livre e Esclarecido, fornecido pelos pesquisadores, baseado nas Diretrizes da Resolução do Conselho Nacional de Saúde (CNS) n 466/2012. A pesquisa teve a aprovação do Comitê de Ética para Pesquisa com Seres Humanos (Parecer n ${ }^{\circ} 84541$ e Certificado de Apresentação de Apreciação Ética (CAAE) no 04654712.0.0000.5188) e foi realizada com base nos determinantes instituídos pelas resoluções no ${ }^{\circ} \mathrm{s}$ 196/1996 e 466/2012 do CNS.

\section{RESULTADOS E DISCUSSÃO}

Ao final da análise, emergiram cinco grandes categorias: insegurança e inexperiência; submissão do paciente; sensação de estar incomodando o paciente; direcionamento de gênero; escolha do paciente por conveniência.

\section{Insegurança e inexperiência}

Essa categoria expressa como o estudante se sente no momento em que realiza os primeiros exames físicos e engloba o conjunto de sentimentos decorrentes da inexperiência e falta de conhecimento técnico inicial, abordando sua insegurança atrelada ao medo e à insegurança de não saber realizá-lo. Isso é o que marca as discussões desse subgrupo, conforme é apresentado no Quadro 1.

$\mathrm{O}$ medo e a insegurança também emergiram no trabalho de Trindade e Vieira ${ }^{4}$, em que cerca de $70 \%$ dos estudantes entrevistados se autodeclararam inseguros, e tal fato acabou os deixando em algumas situações de embaraço e constrangimento perante o paciente ${ }^{4}$. Já Balduino, Palis, Paranaíba, Almeida e Trindade $^{10}$ constataram que $47,3 \%$ dos estudantes participantes raramente se sentem preparados para abordar o paciente, principalmente no que diz respeito às relações interpessoais.

Uma boa abordagem seria ajudar o estudante a perceber suas limitações e integrá-las como algo natural a iniciantes de uma prática clínica. Embora o exame físico seja, de fato, um momento delicado e gerador de ansiedades, ele precisa ser enfrentado com mais tranquilidade, e isso poderia vir dessa compreensão de que é apenas o começo, os primeiros passos da sua longa formação médica ${ }^{11}$.

\section{Submissão do paciente}

Nessa categoria, os alunos externaram uma de suas principais questões: a submissão do paciente dentro do serviço hospitalar, seja em relação aos profissionais em geral ou ao próprio estudante. Nesse contexto, o paciente não tem voz ativa no próprio atendimento. Isso pode ser visto nas falas dos alunos apresentadas no Quadro 2.

Para os alunos, tanto o atendimento quanto a realização do exame demonstram que o paciente não tem o poder de dizer "não" às condutas tomadas pelos profissionais ou estudantes, e, assim, aceita tudo que lhe é proposto.

Isso pode ser visto em pesquisa realizada em uma universidade australiana, cujos resultados mostraram que a maioria dos estudantes entrevistados declarou que os exames físicos não eram para o benefício dos pacientes, mas sim para os objetivos de aprendizado dos alunos ${ }^{12}$. Tal ponto levou à relutância dos participantes em abordar os pacientes, por acreditarem que estes não teriam voz ativa e estariam sempre disponíveis para a realização do exame físico, mesmo quando de teor mais íntimo.

Ao mesmo tempo, dentro do ambiente hospitalar, principalmente em hospitais-escola, os pacientes têm uma posição pouco notada no que diz respeito ao seu poder de voz ativa ${ }^{13}$. Para os autores, o paciente acaba sendo um "material" necessário para o aprender médico, embora não tenha, necessariamente, consciência de sua condição, pois, para ele, o mais importante é a melhora clínica.

Quadro 1
$\begin{gathered}\text { Exemplos de falas que representam a categoria "insegurança e } \\ \text { inexperiência" }\end{gathered}$
"Na teoria você consegue entender como é, mas na prática você não
consegue identificar direito" (Grupo focal 2).
“[...] e outra coisa, a gente mal chegou a fazer um exame, só ela [a
professora] e mais um de nós pôde fazer o exame, ou seja, a gente não sabe
de nada" (Grupo focal 3).
"A gente tem uma aula de duas horas para saber tudo sobre exame
respiratório, mas eu não sei nem o que é para escutar em um exame"
(Grupo focal 6).
“[...] outra coisa importante é o fato de você saber se está fazendo certo ou
não" (Grupo focal 2).

Fonte: Elaborado pelos autores.

\section{Quadro 2 \\ Exemplos de falas que representam a categoria "submissão do paciente"}

“O paciente está numa situação em que ele pode dizer que não quer, mas ele mesmo não tem a consciência. Eu mesma não gostaria que 12 alunos saíssem me auscultando, me apalpando” (Grupo focal 1).

"Ele não tem consciência de que pode dizer que não quer... não tem coragem de dizer não" (Grupo focal 3).

"Pode passar uma ideia de que ele é um rato de laboratório, está sendo explorado" (Grupo focal 6).

“O pior são os comentários: ‘Está hipocorado’ [...] ficam comentando na frente do paciente" (Grupo focal 5).

Fonte: Elaborado pelos autores.

REVISTA BRASILEIRA DE EDUCAÇC̃̃ MÉDICA

3 44 (1) : e027; 2020 


\section{Sensação de estar incomodando o paciente}

Comumente, os estudantes demonstraram preocupação em relação à possibilidade de eles invadirem a privacidade do paciente. Nesse contexto, predominou o sentimento de que a presença e a abordagem dos estudantes, na construção de anamnese e nos exames físicos, gerariam desconforto e incômodo no paciente, deixando-o aborrecido ou constrangido. Isso pode ser observado nas falas manifestadas e impressas no Quadro 3.

Essas falas demonstram um olhar de apreensão dos estudantes quanto à possibilidade de eles invadirem o espaço do paciente, de entrarem na intimidade dele. Tal apreensão ocorre porque, muitas vezes, não se sabe até que ponto o paciente se sente realmente confortável ou não. Esse fato também foi observado em estudo semelhante, no qual os estudantes manifestaram sentimentos de invasão ao abordarem os pacientes, principalmente aqueles que já sofrem por estarem doentes e hospitalizados ${ }^{14}$. Da mesma forma, observou-se que a realização do exame físico, especialmente durante abordagens de natureza íntima, trouxe algumas inquietações à amostra estudada ${ }^{1}$.

Esses sentimentos do estudante perante o paciente são justificados pelo fato de que o exame físico causa um constrangimento natural. Ocorre, nesse momento, certa invasão da intimidade de uma pessoa até então desconhecida; há a exposição do corpo humano, em paradoxal conflito com a noção de pudor e recato socialmente exigida ${ }^{15}$.

Contudo, alguns pacientes têm a percepção de que o estudante está tentando, ao máximo, não o incomodar e acabam sendo mais receptivos, no intuito de melhorar a relação estudante-paciente e torná-la mais leve ${ }^{13}$.

\section{Direcionamento de gênero}

Essa categoria trouxe à tona o impacto do gênero no momento de realização do exame, visto que as pessoas possuem ideologias que transcendem a relação entre estudante e paciente. $O$ exame vai além da técnica, invade aquilo que é ou não permitido na vivência de cada indivíduo. Nesse contexto, destaca-se a forma como o estudante entende as relações de gênero, o que é expresso nas falas do Quadro 4.

Queixas semelhantes são apontadas no que diz respeito aos estudantes que se sentem envergonhados e constrangidos com procedimentos mais íntimos ou quando precisam, de alguma forma, despir o paciente, bem como não se sentem à vontade em examinar pacientes do sexo oposto 4 No mesmo sentido, evidenciou-se que esse desconforto foi maior em estudantes do sexo masculino, o que dificulta o aprendizado ${ }^{12}$.

Dessa forma, é possível notar que a relação de gênero é mais um complicador na relação do estudante com o paciente, pelo constrangimento que pode partir de ambos, de modo que o estudante teme ultrapassar alguma barreira inerente ao paciente, mesmo que essa seja desconhecida, o que prejudica o aprendizado e cultiva o medo no aluno.

\section{Escolha do paciente por conveniência}

Essa categoria demonstra como o aluno pensa no momento de selecionar o paciente para realizar a anamnese e o exame físico, bem como o que leva em consideração para procurar um paciente. Analisemos as falas do Quadro 5.

Assim, a necessidade de conseguir avaliar um paciente que apresente alterações relacionadas ao assunto que está sendo estudado no momento é um dos motivos que predominam, sendo esses pacientes escolhidos, muitas vezes, por possuírem uma doença específica.

\section{Quadro 3 \\ Exemplos de falas que representam a categoria "sensação de estar incomodando o paciente"}

"Você pensa que você é inconveniente ali naquele momento, mas tem que pensar também que daqui a duas horas vai vir outro estudante e vai repetir tudo de novo" (Grupo focal 4).

"Eu me sentiria incomodado. Eu, doente, e vindo 1, 2, 3, 4, 5 pessoas me examinar [...]. Meia hora vêm mais 3 pessoas" (Grupo focal 2).

"Ninguém se liga que ali é uma pessoa, que tem vergonha, que tem medos [...] que não quer que alguém mexa com ele, que fique palpando" (Grupo focal 4).

“[...] tinha uma paciente com ICC que não tava nem conseguindo respirar direito, aí todos os grupos de semio resolveram fazer ausculta naquela mulher [...] respira, inspira, expira [...] aquilo me incomodou muito" (Grupo focal 5).

Fonte: Elaborado pelos autores.

\section{Quadro 4 \\ Exemplos de falas que representam a categoria "direcionamento de gênero" \\ "A questão de gênero também é importante, tipo eu sou homem e não sei se uma paciente vai querer que eu a examine" (Grupo focal 3 ). \\ "Quando estamos só homens, querendo ou não, a paciente mulher tem direito de ficar desconfortável com a gente" (Grupo focal 6). \\ "Para o exame físico, eu prefiro escolher homem porque vai ter que tirar a camisa em algum momento" (Grupo focal 1).}

"Para a mulher é mais fácil do que para o homem [...] uma mulher consegue fazer exame físico em outra mulher ou em um homem [...]" (Grupo focal 2).

Fonte: Elaborado pelos autores.

Quadro 5
Exemplos de falas que representam a categoria “escolha do paciente
por conveniência”
“[...] outra coisa, a gente sempre tende a procurar um paciente mais
tranquilo para fazer o exame físico... nunca ninguém faz com um acamado,
por exemplo" (Grupo focal 4).
"No início, quando era só anamnese, a gente procurava um paciente que
estivesse disposto a conversar, aí agora a gente já vai mais direcionado [...]
quem é o paciente da pneumologia ou da cardiologia [...]” (Grupo focal 5).
“[...] um paciente acamado, mais complicado [...] é muito difícil, até para
que você não cometa muitos erros" (Grupo focal 2).

Fonte: Elaborado pelos autores.

Além disso, a personalidade e a disponibilidade do paciente em ofertar ou não o seu tempo ao estudante contam muito na escolha deste. Tal fato foi observado em um estudo em que 20,3\% dos estudantes participantes demonstraram ter dificuldades em encontrar o paciente "ideal", ou seja, aquele que estivesse disposto a facilitar a realização da anamnese e do exame físico, bem como sentiam-se angustiados em fazer essa busca ${ }^{3}$. 


\section{CONCLUSÕES}

Verifica-se que a insegurança, a inexperiência e a sensação de estar utilizando o paciente como um objeto são percepções que geram desconforto nos estudantes e dificultam seu aprendizado.

Além disso, os alunos se preocupam com a situação dos pacientes que são, frequentemente, expostos e avaliados por eles. Entendem que o paciente pode sentir que seu espaço foi invadido e sabem que ele, muitas vezes, não tem a liberdade de dizer se gostaria ou não de ser examinado por estudantes e professores.

Tais percepções podem limitar o aprendizado quando são vivenciadas sem proporções maiores e irrealísticas. Ao mesmo tempo, quando acolhidas e usadas de forma favorável à formação médica, não impedem a realização do exame e podem fortalecer, no estudante, a atenção ao vínculo e um maior o cuidado com o outro.

Desse modo, conhecer as reações do estudante que são inerentes ao enfrentamento na formação médica pode favorecer a construção de habilidades médicas de forma mais saudável para o aluno e positivas para a relação estudante-paciente, apresentando-se, portanto, como um campo importante de pesquisa médica direcionada a oferecer recursos que promovam a melhoria do ensino-aprendizagem.

\section{REFERÊNCIAS}

1. Muñoz RL de S, Silva IBA, Maroja JLS. Medical students' experience in practical classes on bedside clinical examination. Rev. bras. educ. méd. 2011;35(3):376-81.

2. Kiyoshi T, Braga K. The teaching of clinical skills and the applicability of a simplified guide for physical examination in the undergraduate medical program. Rev. bras. educ. méd. 2017; 41(2):299-309.

3. Paiva GPO, Herculano TB, Gama ALH, Cabral RP, Campos DB, Oliveira DNS de. Enfrentamentos do estudante na iniciação da Semiologia Médica. Rev. bras. educ. méd. 2018;42(2): 79-88.

4. Trindade LMDF, Vieira MJ. Medicine students and coping strategies in patient care. Rev. bras. educ. méd. 2013;37(2):167-77 [acesso em 29 out 2018]. Disponível em: http://www.scielo.br/pdf/rbem/v37n2/03.pdf.

5. Mascia AR, Silva FB, Lucchese AC, Marco MA de, Cezira M, Nogueira F et al. Prática médica: estudo transversal. Education 2009;33(1):40-8.

6. Mori MO, Valente TC, Nascimento LFC. Síndrome de burnout e rendimento acadêmico em estudantes da primeira à quarta série de um curso de graduação em Medicina. Rev. bras. educ. méd. 2012;36(4):536-40.

7. Universidade Federal da Paraíba. Coordenação do Curso de Graduação em Medicina. Projeto Político-Pedagógico do Curso de Graduação em Medicina. João Pessoa: UFPB, CCS, Coppem; 2007.

8. Bardin L. Análise de conteúdo. 5. ed. São Paulo: Edições 70; 2011.

9. Silva AH, Trevisan, Fossa MI. Análise de conteúdo: exemplo de aplicação da técnica para análise de dados qualitativos. Qualitas 2015;16(1):1-14.
10. Balduino PM, Palis FP, Paranaíba VF, Almeida HO de, Trindade EMV. A perspectiva do paciente no roteiro de anamnese: o olhar do estudante. Rev. bras. educ. méd. 2012;36(3):335-42.

11. Camacho NCA, Leone OD, Cataldo Neto A. O estudante de medicina e seus pacientes. 3. ed. Porto Alegre: EDIPUCRS; 2018.

12. Dabson AM, Magin PJ, Heading G, Pond D. Medical students' experiences learning intimate physical examination skills: a qualitative study. BMC Med Educ 2014;14(1):1-7.

13. Dorigatti AE, Faber EW, Garcia Junior C, Turato ER. Como se sentem pacientes quando examinados por estudantes de Medicina? Um misto entre ambiguidades e satisfações encontradas em estudo qualitativo. Rev. bras. educ. méd. 2015;39(1):95-101.

14. Claro LBL, Mendes AAA. Uma experiência do uso de narrativas na formação de estudantes de medicina. Interface (Botucatu) 2018;22(65):621-30.

15. Dichi JB, Dichi I. Agonia da história clínica e suas conseqüências para o ensino médico. Rev. bras. educ. méd. 2007;30(2):93-7.

\section{CONTRIBUIÇÃO DOS AUTORES}

Gilka Paiva Oliveira Costa: concepção e planejamento do projeto de pesquisa, análise dos dados, redação e revisão crítica do manuscrito e aprovação da versão submetida à revista.

Kaio Alighieri Nunes de França: concepção e planejamento do projeto de pesquisa, coleta e análise dos dados, redação e revisão crítica do manuscrito e aprovação da versão submetida à revista.

Maria Albertina Leite Santos e Janaína Guerra Guilherme: coleta e discussão dos dados, redação e revisão crítica do manuscrito e aprovação da versão submetida à revista.

José Givaldo Melquiades de Medeiros e Estácio Amaro da Silva Júnior: redação e revisão crítica do manuscrito e aprovação da versão submetida à revista.

\section{CONFLITO DE INTERESSES}

Os autores declaram não haver conflito de interesses neste estudo.

\section{ENDEREÇO PARA CORRESPONDÊNCIA}

Kaio Alighieri Nunes de França. Centro de Ciências Médicas/ Departamento de Medicina Interna, $1^{\circ}$ andar, Rua Tabelião Stanislau Eloy, 831, Cidade Universitária, João Pessoa, PB, Brasil, CEP: 58050-585.

E-mail: kaionuunes@gmail.com 